\title{
Phase-field-lattice Boltzmann study for lamellar eutectic growth in a natural convection melt
}

\author{
Ang Zhang', Zhi-peng Guo', and *Shou-mei Xiong ${ }^{1,2}$ \\ 1. School of Materials Science and Engineering, Tsinghua University, Beijing 100084, China \\ 2. Key Laboratory for Advanced Materials Processing Technology, Ministry of Education, Tsinghua University, Beijing 100084, China
}

\begin{abstract}
In the present study, the influence of natural convection on the lamellar eutectic growth is determined by a phase-field-lattice Boltzmann study for Al-Cu eutectic alloy. The mass difference resulting from concentration difference led to the fluid flow, and a robust parallel and adaptive mesh refinement algorithm was employed to improve the computational efficiency without any compromising accuracy. Results show that the existence of natural convection would affect the growth undercooling and thus control the interface shape by adjusting the lamellar width. In particular, by alternating the magnitude of the solute expansion coefficient, the strength of the natural convection is changed. Corresponding microstructure patterns are discussed and compared with those under no-convection conditions.
\end{abstract}

Key words: natural convection; lamellar width; phase field model; lattice Boltzmann method
CLC numbers: TP391.99
Document code: A
Article ID: 1672-6421(2017)05-373-06

\begin{abstract}
$\mathrm{A}$ one of the most important solidification patterns, eutectic microstructure has been recognized for its significant influence on the ultimate mechanical properties of the materials ${ }^{[1,2]}$. Since the classical mathematical analysis for the steady-state growth of eutectic alloy was presented by Jackson and Hunt ${ }^{[3]}$, theoretical studies on eutectic solidification have attracted increasing attention.

However, the influence of convection on eutectic growth still lacks sufficient research. Generally, there are two main types of convection considered during the directional solidification of eutectic alloys, i.e., forced convection caused by imposed external fields, and natural convection driven by the solute (or density) difference. Considering that there are many uncertainties in experiments, numerical simulation has become an indispensable way to accurately recover the underlying physics during solidification ${ }^{[4-7]}$. Wang et al. ${ }^{[8]}$ performed a Monte-Carlo simulation of eutectic growth with weak convection and found that convection made
\end{abstract}

\section{*Shou-mei Xiong}

Male, Ph. D. Professor. Research interests: Al and Mg alloys high pressure die casting and microstructure simulation during solidification.

E-mail: smxiong@tsinghua.edu.cn

Received: 2017-08-28; Accepted: 2017-09-11 the distribution of lamellar spacing more scattered. But beyond that, to the best of our knowledge, there has been no more numerical case to study the effect of convection on eutectic growth.

Encouragingly, a robust parallel-adaptive mesh refinement algorithm (Para-AMR) has been developed to efficiently solve the phase field equations including the dendritic ${ }^{[9]}$ and eutectic transitions ${ }^{[10]}$. The lattice Boltzmann method (LBM) has also emerged with great potential to numerically solve the energy, momentum and mass transport problems by a relaxation to a local equilibrium ${ }^{[11-13]}$. Therefore, it seems reasonable to couple the LBM into eutectic phase-field models to retrieve the interaction of eutectic microstructure and flows.

In this work, we coupled the LBM into the developed Para-AMR algorithm to reveal the effect of natural convection on eutectic growth. The phase field model was employed to simulate eutectic evolution while the LBM was for the simultaneous calculation of melt convection. The velocity field near the solid/liquid (S/ L) interface was explored and several key mechanisms were highlighted. In particular, by alternating the magnitude of solute expansion coefficient, the solute distribution and the microstructure patterns were discussed, especially the width of solid phases closely related to the interface undercooling. 


\section{Methods}

\subsection{Phase field model}

The phase model proposed by Kim et al. ${ }^{[14]}$ was adopted in this work. By employing three order parameters $\phi_{1}, \phi_{2}$ and $\phi_{3}$ to denote the liquid phase (L) and two solid phases $(\alpha$ and $\beta)$, the energy functional $F$ and corresponding governing equations are:

$F=\int_{V}\left[\sum_{j>i} \sum_{i}\left(-\frac{\varepsilon_{i j}^{2}}{2} \nabla \phi_{i} \cdot \nabla \phi_{j}+\omega_{i j} \phi_{i} \phi_{j}\right)+\sum_{i} \phi_{i} f^{i}\left(c_{i}\right)+\lambda_{\mathrm{L}}\left(\sum_{i} \phi_{i}-1\right)\right] d V$

$$
\begin{gathered}
\frac{\partial \phi_{i}}{\partial t}=-\frac{2}{n} \sum_{j \neq i}^{n} s_{i} s_{j} M_{i j}\left(\frac{\delta F}{\delta \phi_{i}}-\frac{\delta F}{\delta \phi_{j}}\right) \\
\frac{\partial C}{\partial t}+\phi_{3} \vec{v} \cdot \nabla C=\nabla \cdot D \sum_{i} \phi_{i} \nabla C_{i}
\end{gathered}
$$

where the coexisting three phases $\phi_{i}(i=1,2,3)$ vary between 0 and 1 to maintain the sum to be 1 at any position, e. g., $\phi_{3}=1$ and $\phi_{1}$ $=\phi_{2}=0$ in L phase, and $\lambda_{\mathrm{L}}$ is the corresponding Lagrange multiplier. $\varepsilon_{i j}$ is the gradient energy coefficient, $\omega_{i j}$ is the height of the double well potential and $s_{i}$ is a step function, i.e., $s_{i}(x, t)=0$ if $\phi_{i}=0$ and $s_{i}(x, t)=1$ otherwise. $M_{i j}$ is the phase-field mobility and determined by assuming a vanishing kinetic effect during solidification. The composition of the coexisting phases is determined by a weighted average, i.e., $C(x, t)=\sum \phi_{i} C_{i} . \vec{v}$ is the intrinsic flow velocity induced by the concentration difference, which is expanded as $\vec{v}=(u, v)$ in 2D case and calculated using the LBM. $D$ is the diffusivity and is determined by the linear interpolation between the solid and liquid phases.

$$
D=\phi_{3} \cdot D_{\mathrm{L}}+\left(1-\phi_{3}\right) \cdot D_{\mathrm{s}}
$$

where $D_{\mathrm{L}}$ and $D_{\mathrm{S}}$ are the diffusivity of liquid and solid phases respectively.

\subsection{Lattice Boltzmann method}

As a mesoscopic kinetic-based approach, the LBM assumes that the flow field is comprised of a series of pseudo-particles represented by a distribution function ${ }^{[15]}$. The macroscopic flow is characterized by the streaming and collision of these quasiparticles. In the current model, a so-called two-dimensional nine-velocity (D2Q9) model was employed to solve the natural convection. The discrete velocities $c_{i}$ along nine different directions are defined as:

$$
c_{i}= \begin{cases}(0,0) & i=0 \\ c\left(\cos \left((i-1) \frac{\pi}{2}\right), \sin \left((i-1) \frac{\pi}{2}\right)\right) & i=1,2,3,4 \\ \sqrt{2} c\left(\cos \left((2 i-1) \frac{\pi}{4}\right), \sin \left((2 i-1) \frac{\pi}{4}\right)\right) & i=5,6,7,8\end{cases}
$$

where $c=\delta x / \delta t$ is the lattice velocity, $\delta x$ is the lattice spacing, equal for both directions, and $\delta t$ is the time step. The evolution equation when considering the discrete force $G_{i}(x, t)$ is expressed as:

$$
f_{i}\left(\vec{r}+c_{i} \delta t, t+\delta t\right)=f_{i}(\vec{r}, t)-\frac{1}{\tau}\left(f_{i}(\vec{r}, t)-f_{i}^{e q}(\vec{r}, t)\right)+G_{i}(\vec{r}, t) \delta t
$$

where $f_{i}(\vec{r}, t)$ is the particle distribution function in the $i$-th direction, which represents the possibility of finding a pseudoparticle at the position $\vec{r}$ and time $t$. $\tau$ is the single relaxation time related to the kinematic viscosity, i.e., $v=c^{2} \delta t(2 \tau-1) / 6 . f_{i}^{e q}(\vec{r}, t)$ is the equilibrium distribution function and expressed as:

$$
f_{i}^{e q}=\rho w_{i}\left(1+\frac{3 \vec{c}_{i} \cdot \vec{v}}{c^{2}}+\frac{9\left(\vec{c}_{i} \cdot \vec{v}\right)^{2}}{2 c^{4}}-\frac{3 \vec{v} \cdot \vec{v}}{2 c^{2}}\right)
$$

where $\rho=\sum_{i} f_{i}$ is the local fluid density, $\vec{v}=\sum_{i} f_{i} \vec{c}_{i} / \rho$ is the flow velocity and $w_{i}$ is the weight coefficient determined by the discrete velocity model, i.e., $w_{0}=4 / 9, w_{1-4}=1 / 9$, and $w_{5-8}=1 / 36$ for the D2Q9-lattice velocity model. The discrete force $G_{i}(x, t)$ with second-order accuracy is given by:

$$
\begin{gathered}
G_{i}=\rho w_{i}\left(3 \frac{\vec{c}_{i}-\vec{v}}{c^{2}}+\frac{9\left(\vec{c}_{i} \cdot \vec{v}\right) \vec{c}_{i}}{2 c^{4}}\right) \cdot\left(G_{D}+G_{B}\right) \\
G_{D}(\vec{r}, t)=-\frac{2 \rho v h}{W_{0}}\left(1-\phi_{3}\right)^{2} \vec{v} \\
G_{B}(\vec{r}, t)=-\rho \vec{g} \beta_{c}\left(C-C_{0}\right) \phi_{3}
\end{gathered}
$$

where $G_{D}$ is the dissipative drag force in the vicinity of the $\mathrm{S} / \mathrm{L}$ interface to satisfy the no-slip boundary condition ${ }^{[16]} . h=2.757$ is a dimensionless constant obtained by an asymptotic analysis of plane flow past the diffusive interface ${ }^{[17]}$. $W_{0}$ is the interface thickness, and $G_{B}$ is the buoyancy force induced by the concentration difference in the liquid ${ }^{[18]} \cdot \vec{g}$ is the gravitational acceleration, $\beta_{c}$ is the solute expansion coefficient and $C_{0}$ is the initial liquid concentration.

\subsection{Numerical approach}

The Para-AMR algorithm with block structure adaptive mesh refinement and parallel computing scheme was employed to solve the phase-field-lattice Boltzmann equations. The details of this algorithm have been illustrated in References [9, 10] and only main features are summarized here.

The refinement process was proceeded first by tagging the potential grids according to a predefined gradient criterion,

$$
\max _{1 \leq i \leq 3}\left|\nabla \phi_{i}\right|+E_{c}|\nabla C|+E_{v}\left(\sqrt{|\nabla u|^{2}+|\nabla v|^{2}}\right) \geq \xi
$$

where $E_{c}$ and $E_{v}$ are weighted coefficients for solute and velocity, respectively, and $\xi$ is a threshold value determined via numerical tests. $u$ and $v$ are the two axial velocity components, i.e., $\vec{v}=(u, v)$. The $\mathrm{S} / \mathrm{L}$ interface during solidification is the position where the gradient reaches the local maximum and thus mesh refinement is needed. After constructing a hierarchical architecture with different sets of patch-boxes on each grid level, the local data was then broadcasted to all processors to realize the parallel computation. 


\subsection{Material and initial condition}

All simulations were performed under a constant undercooling $1 \mathrm{~K}$ using Al-Cu eutectic alloy, whose physical parameters ${ }^{[18-21]}$ are provided in Table 1 . It should be noted that the solute expansion coefficient $\beta_{c}$ was negative, which indicates that the larger the concentration, the heavier the system ${ }^{[18]}$. The initial eutectic composition was set to be eutectic point, i.e., 0.173 mol. frac., and the minimum mesh size $d x_{\min }$ was $0.2 \mu \mathrm{m}$. The time step was computed as:

$$
d t=0.8 \cdot \frac{d x_{\min }^{2}}{2 \times N_{d} \times D_{\mathrm{L}}}
$$

where $N_{d}=2$ is the dimension of the domain. Four eutectic couples with the height of $3 \mu \mathrm{m}$ were initialized at the bottom of the computational domain, whose size was $51.2 \times 51.2$ $\mu \mathrm{m}^{2}$. The initial eutectic lamellar spacing was computed as $6.4 \mu \mathrm{m}$ according to the theoretical analysis by Datye and Langer ${ }^{[22]}$. A periodic boundary condition was set at the left and right boundaries for all variables to simulate an infinite melt reservoir. For the bottom and top boundary conditions, an adiabatic condition was for the phase field variable, while a no-slip condition was for the flow field variable.

Table 1: Physical parameters of Al-Cu eutectic alloy

\begin{tabular}{lc}
\multicolumn{1}{c}{ Parameter } & Value \\
\hline$D_{\mathrm{L}}$ (diffusivity in liquid, $\left.\mathrm{m}^{2} \cdot \mathrm{s}^{-1}\right)$ & $3 \times 10^{-9}$ \\
$D_{\mathrm{S}}$ (diffusivity in solid, $\left.\mathrm{m}^{2} \cdot \mathrm{s}^{-1}\right)$ & $3 \times 10^{-13}$ \\
$m_{\alpha}$ (liquidus slope of $\alpha$ phase at eutectic temperature, $\mathrm{K} \cdot(\mathrm{mol} \text { frac. })^{-1}$ & -1050 \\
$m_{\beta}$ (liquidus slope of $\beta$ phase at eutectic temperature, $\mathrm{K} \cdot(\mathrm{mol} \text { frac. })^{-1}$ & 488 \\
$k_{\alpha}$ (partition coefficient in $\alpha$ phase to the liquid phase) & 0.1445 \\
$k_{\beta}$ (partition coefficient in $\beta$ phase to the liquid phase) & 1.85 \\
$T_{\mathrm{E}}$ (eutectic temperature, $\left.\mathrm{K}\right)$ & 821.4 \\
$\sigma_{\alpha L}\left(\alpha / \mathrm{L}\right.$ interface energy, $\left.\mathrm{J} \cdot \mathrm{m}^{-2}\right)$ & $160.01 \times 10^{-3}$ \\
$\sigma_{\beta L}\left(\beta / \mathrm{L}\right.$ interface energy, $\left.\mathrm{J} \cdot \mathrm{m}^{-2}\right)$ & $88.363 \times 10-3$ \\
$\sigma_{\alpha \beta}\left(\alpha / \beta\right.$ interface energy, $\left.\mathrm{J} \cdot \mathrm{m}^{-2}\right)$ & $219.484 \times 10^{-3}$ \\
$g$ (gravitational acceleration, $\left.\mathrm{m} \cdot \mathrm{s}^{-1}\right)$ & 9.8 \\
$\beta_{c}$ (solute expansion coefficient) & $-7.3 \times 10^{-3}$
\end{tabular}

\section{Results}

Figure 1 shows the typical solute field and velocity field of Al$\mathrm{Cu}$ eutectic alloy. The velocity vector in front of the growing interface is from $\alpha$ to $\beta$ phase, as designated by the red arrows in Figs. 1(a) and (c). The contour maps of the velocity $\vec{v}=(u, v)$ are shown in Figs. 1(d) and (f), in which the darker the color, the greater the value, and the red arrows point to the direction of the increasing velocity. Both the transverse and longitudinal velocity contour maps are almost symmetric at about the triple point.

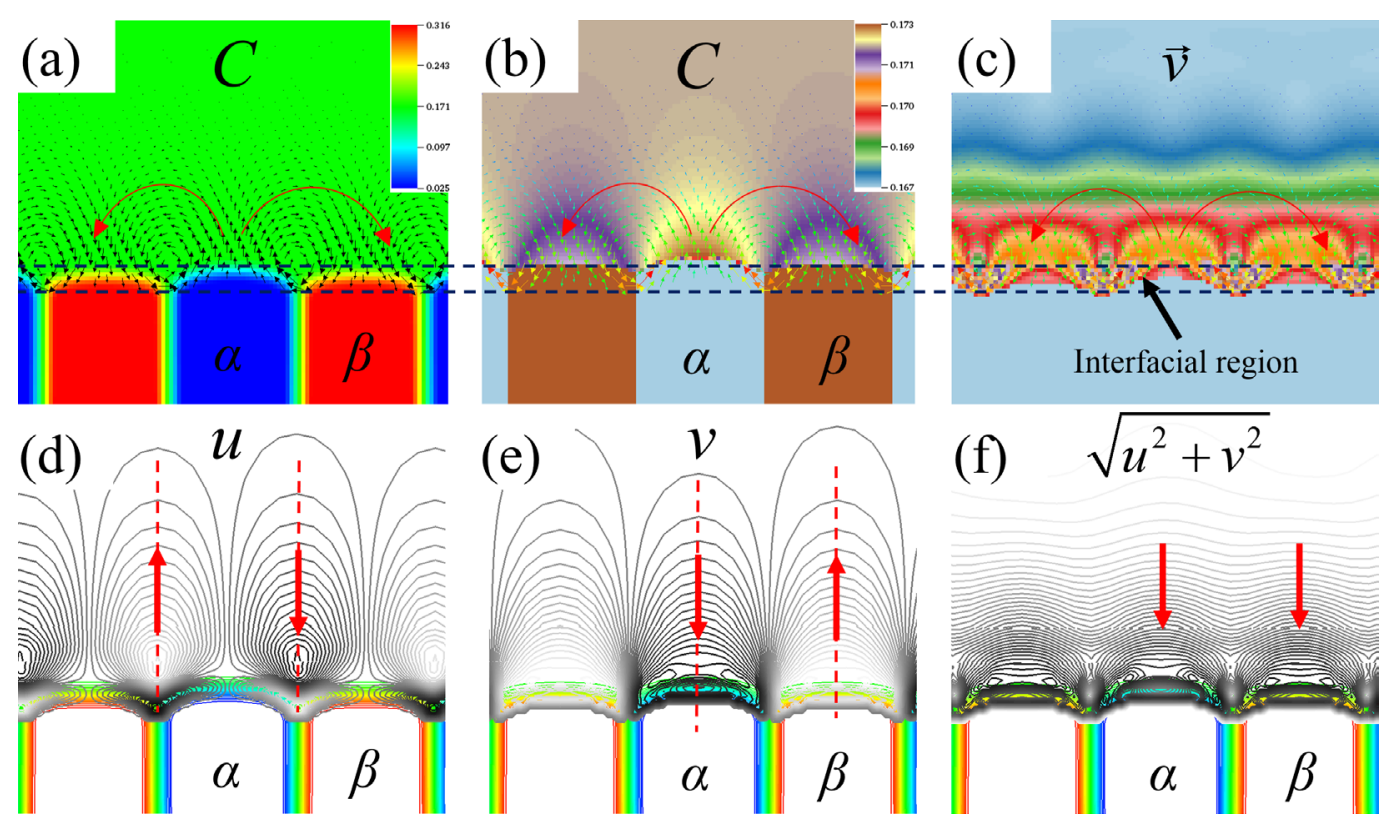

Fig. 1: Solute field and velocity field of Al-Cu eutectic alloy 
Figure 2 shows the distribution of the solute concentration along the given directions (i.e., $V_{1}$ and $V_{2}$ ) perpendicular to the $\mathrm{S} / \mathrm{L}$ interface. $C_{\alpha \mathrm{L}}$ and $C_{\beta \mathrm{L}}$ are the liquid concentration in equilibrium with $\alpha$ and $\beta$ phase at temperature $T$, respectively. As the distance increased, the solute concentration changed monotonically until reaching a constant, as indicated by the dot line in Fig. 2. Furthermore, the concentration difference $C-C_{0}$ was converted to the mass difference by multiplying the solute expansion coefficient $\beta_{c}$ [see Eq. (8c)]. A negative $\beta_{c}$ indicates that the larger the concentration, the heavier the liquid phase ${ }^{[18]}$. Specifically, for Al-Cu alloy with negative $\beta_{c}$, a larger concentration means that the liquid has more $\mathrm{Cu}$ atoms and thus will be heavier. $C_{\alpha \mathrm{L}}$ is larger than $C_{\beta \mathrm{L}}$ and thus the liquid concentration near $\alpha / \mathrm{L}$ interface is heavier for $\mathrm{Al}-\mathrm{Cu}$ alloy. As the distance away from the $\mathrm{S} / \mathrm{L}$ interface increases, the concentration near $\alpha / \mathrm{L}$ interface decreases while that near $\beta / \mathrm{L}$ interface increases until approaching the far-field concentration (i.e., the initial concentration $C_{0}$ ). Accordingly, as the distance increases, the liquid near $\alpha / \mathrm{L}$ interface will become increasingly lighter due to less $\mathrm{Cu}$ atoms, which is just the opposite for that near $\beta / \mathrm{L}$ interface. It should be noted that the solute concentration near $\alpha / \mathrm{L}$ interface is always higher than that near $\beta / \mathrm{L}$ interface and thus the liquid near $\alpha / \mathrm{L}$ interface is heavier for Al-Cu alloy. Because the buoyancy force induced by the mass difference will drive the liquid flow into the lighter region, there will be an outflow from $\alpha$ phase and an inflow to $\beta$ phase, as shown in Fig. 1(a).

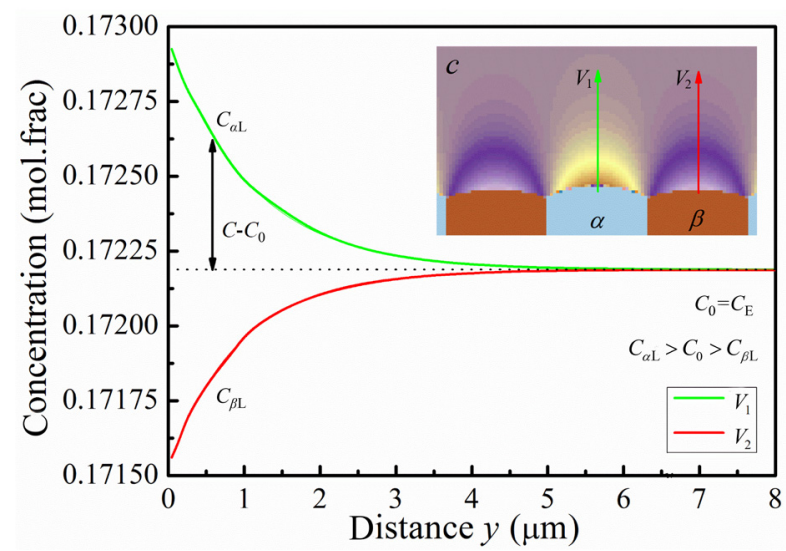

Fig. 2: Distribution of solute concentration along given directions perpendicular to $S / L$ interface

Figure 3 shows the velocity distribution along the direction parallel to the $\mathrm{S} / \mathrm{L}$ interface at $y=0.05 S_{\alpha \beta}\left(S_{\alpha \beta}\right.$ $=\lambda / 2, \lambda$ is lamellar spacing) away from the interface, and the minus velocity indicates that the velocity is along the negative $y$-direction. The velocity- $x$ curves, including transverse velocity, longitudinal velocity and sum velocity, all presented symmetry patterns about the centerline of solid phase, which agreed well with the velocity contour map in Fig. 1. The transverse velocity reached its maximum at the boundary between $\alpha$ and $\beta$ phases, while the maximum of the longitudinal velocity was in the middle of the solid phases.

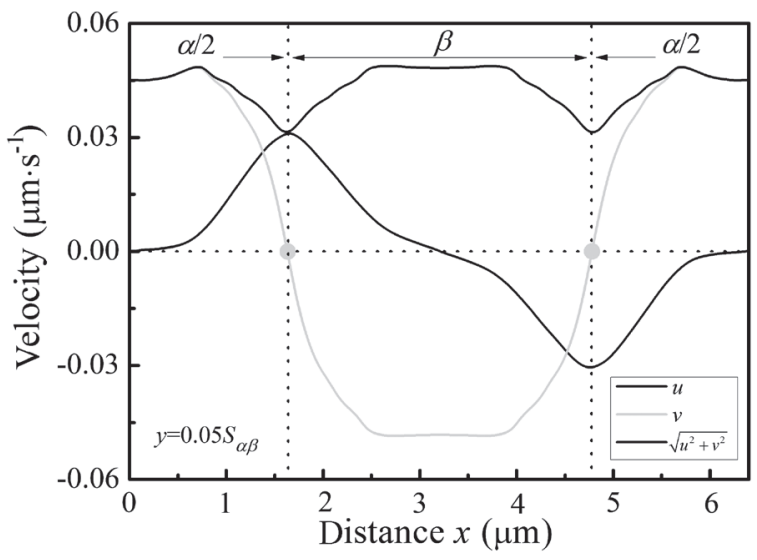

Fig. 3: Velocity distribution along the direction parallel to $S / L$ interface

\section{Discussion}

Natural convection has inevitable effects on the concentration field and thus will change the interfacial solute undercooling $\Delta T_{c}$. Figure 4 shows the solute concentration of $\mathrm{Al}-\mathrm{Cu}$ alloy at the specific $\alpha / \mathrm{L}$ interface (i.e., $\phi_{1}=\phi_{3}=0.5$ ). To thoroughly investigate the effect of the buoyancy force, the solute expansion coefficient varied from $10^{-4} \beta_{c 0}$ to $10^{3} \beta_{c 0}$ in units of $\beta_{c 0}= \pm 7.3 \times 10^{-3}$ [21]. With increasing effects of the buoyancy force, the concentration near $\alpha / \mathrm{L}$ interface deviated increasingly from that under no-convection condition, and accordingly, the interfacial solute undercooling $\Delta T_{c}$ would change to remain compatible with this variation.

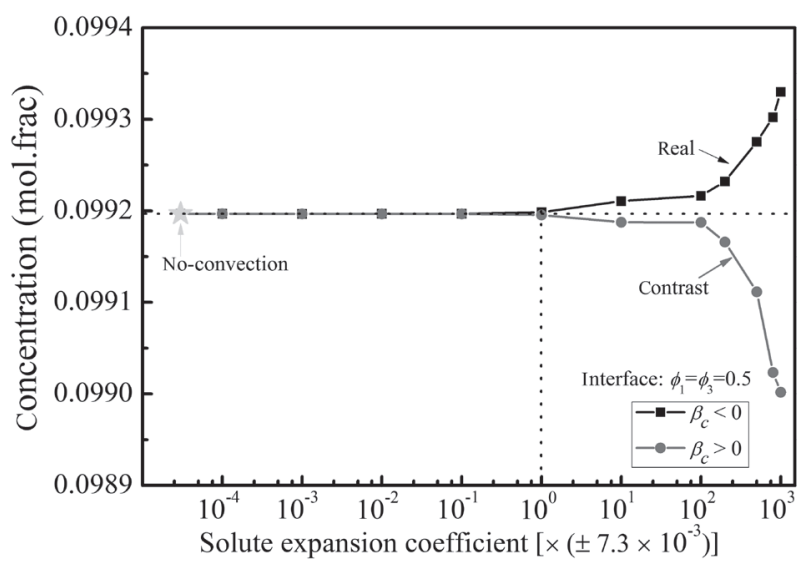

Fig. 4: Solute concentration of Al-Cu alloy at specific $\alpha / L$ interface

The temperature field is imposed by the macroscopic heat fluxes, e.g., assumed to be isothermal with constant undercooling $(\Delta T=1 \mathrm{~K})$ in this work. To compensate for the difference between the imposed undercooling $\Delta T$ and the $\mathrm{S} / \mathrm{L}$ interfacial solute undercooling $\Delta T_{c}$, as indicated by Eq. (11), the shape of the $\mathrm{S} / \mathrm{L}$ interface has to be changed by adjusting the local curvature.

$$
\Delta T=\Delta T_{c}+\Delta T_{r}=m\left(C_{E}-C(x)\right)+\Gamma \kappa(x)
$$

where, the kinetic undercooling was reasonably ignored ${ }^{[3]}$ and 
$\Delta T_{r}=\Gamma \bullet \kappa(x)$ is the curvature undercooling, $m$ is the liquidus slope, $C_{E}$ and $C(x)$ are the eutectic and interfacial composition, respectively. $\Gamma$ is the Gibbs-Thompson coefficient and $\kappa(x)$ is the local interfacial curvature.

When the solute expansion coefficient was negative (i.e., the real value for $\mathrm{Al}-\mathrm{Cu}$ alloy), the flow direction was from $\alpha$ to $\beta$ phase and thus large numbers of solute atoms near $\alpha / \mathrm{L}$ interface were timely transported into the far-field liquid, which contributed to the solute precipitation of $\alpha$ phase. Accordingly, both the interfacial solute concentration and concentration difference with the initial concentration became larger, which resulted in the increase of the interfacial solute undercooling $\Delta T_{c}$. Thus, the curvature undercooling $\Delta T_{r}$ needed to be diminished to maintain an isothermal $\alpha / \mathrm{L}$ interface.

Assuming that the real interface shape is described by the relation $y=I(x)$, the average curvature of $\alpha / \mathrm{L}$ interface is given by:

$$
\left\langle\kappa_{\alpha}(x)\right\rangle=\frac{1}{S_{\alpha}} \int_{0}^{S_{\alpha}} \kappa_{\alpha}(x) d x=\frac{1}{S_{\alpha}} \int_{0}^{S_{\alpha}} \frac{-d^{2} I / d x^{2}}{\left(1+(d I / d x)^{2}\right)^{3 / 2}} d x=\frac{\sin \theta_{\alpha}}{S_{\alpha}}
$$

where $S_{\alpha}$ is half the width of $\alpha$ phase, and $\theta_{\alpha}$ is the contact angle at the $\alpha / \mathrm{L}$ interface and assumed constant. Therefore, the curvature undercooling $\Delta T_{r}$ is actually changed by only adjusting the width of solid phases, i.e., $S_{\alpha}$ and $S_{\beta}$. As shown in Fig. 5, the width of solid phases presented an obvious trend with increasing solute expansion coefficient, and had opposite change for positive and negative $\beta_{c}$.

With the increasing half width of $\alpha$ phase $\left(S_{\alpha}\right)$, the half width of $\beta$ phase $\left(S_{\beta}\right)$ continued to decline for negative $\beta_{c}$, while the lamellar spacing $\lambda$, i.e., $2\left(S_{\alpha}+S_{\beta}\right)$, remained almost constant during all simulations, as shown in Fig. 6. The effect of the natural convection on the eutectic lamellar spacing was quite small in an alloy of eutectic composition, which agreed well with experimental results by Lee, et $\mathrm{al}^{[23]}$.

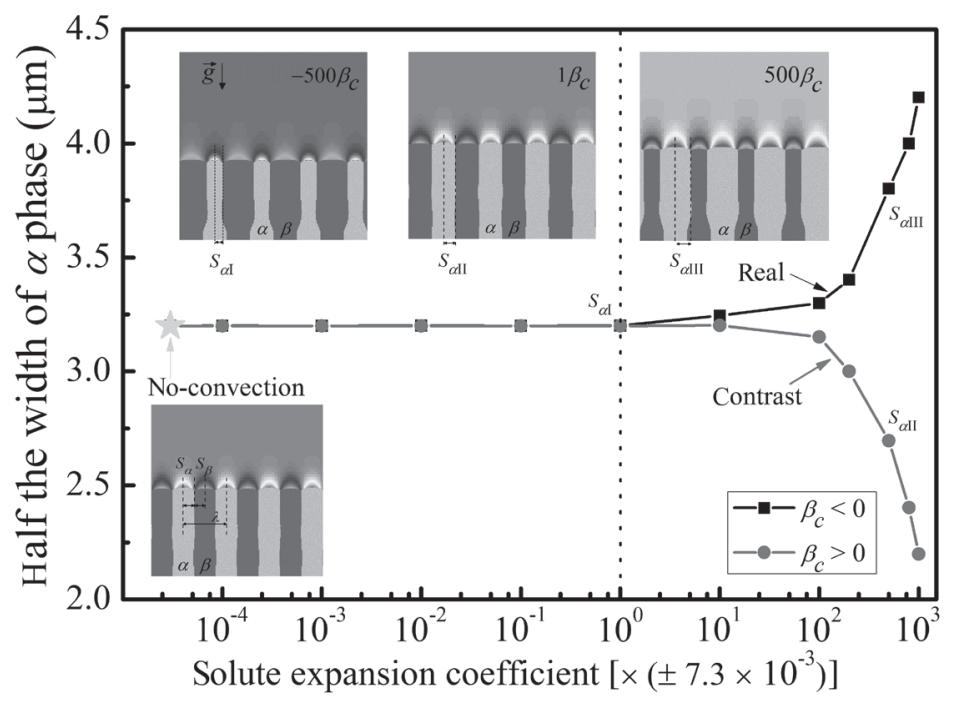

Fig. 5: Half the width of $\alpha$ phase varying with solute expansion coefficient

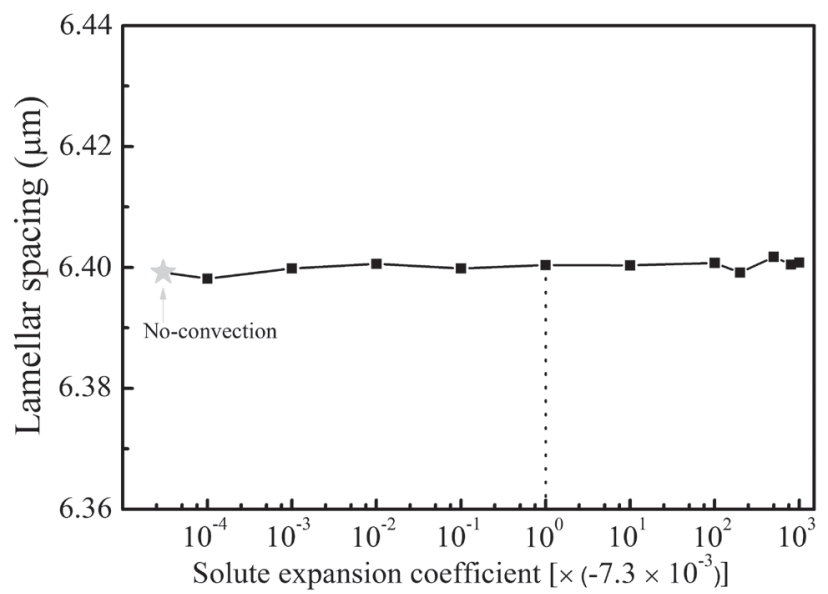

Fig. 6: Lamellar spacing varying with solute expansion coefficient

\section{Conclusions}

The LBM was coupled into the eutectic phase-field model to simulate the eutectic growth under natural convection. To improve the computation efficiency, the Para-AMR algorithm was employed to reveal the underlying physics without any compromising accuracy. Results show that the transverse and longitudinal velocities have different trends, and with increasing solute expansion coefficient, the width of solid phases will have corresponding adjustment, e.g., increasing for $\alpha$ phase but decreasing for $\beta$ phase, to maintain the stability of the interface, while the eutectic lamellar spacing stays constant in an alloy of eutectic composition.

\section{References}

[1] Dantzig J A, Rappaz M. Solidification. Lausanne: EPFL Press, 2009.

[2] Akamatsu S, Plapp M. Eutectic and peritectic solidification patterns. Current Opinion in Solid State and Materials Science, 2016, 20(1): 46-54.

[3] Jackson K A, Hunt J D. Lamellar and rod eutectic growth. Trans. Metall. Soc. AIME, 1966, 236: 11291142.

[4] Xu Qingyan, Feng Weiming, Liu Baicheng, et al. Numerical simulation of dendrite growth of aluminum alloy. Acta Metall. Sin., 2002, 38(8): 799-803. (In Chinese)

[5] Fu Zhen-Nan F, Xu Qing-Yan, Xiong Shou-Mei. Numerical simulation on dendrite growth process of Mg alloy using cellular automaton method based on probability capturing model. Chin. J. Nonferrous Met., 2007, 17(10): 1567-1573. (In Chinese)

[6] Guo Z, Mi J, Xiong S, et al. Phase field simulation of binary alloy dendrite growth under thermal- and forcedflow fields: an implementation of the parallel-multigrid approach. Metall. Mater. Trans. B, 2013, 44(4): 924937.

[7] Yang M, Xiong S M, Guo Z. Characterisation of the 3-D dendrite morphology of magnesium alloys using synchrotron X-ray tomography and 3-D phase-field 
modelling. Acta Mater., 2015, 92: 8-17.

[8] Wang W M, Liu Z G, Liu J M, et al. Eutectic patterns with weak convection in binary systems. J. Cryst. Growth, 2002, 240(1): 313-320.

[9] Guo Z, Xiong S M. On solving the 3-D phase field equations by employing a parallel-adaptive mesh refinement (Para-AMR) algorithm. Comput. Phys. Commun., 2015, 190: 89-97.

[10] Zhang A, Guo Z, Xiong S M. Eutectic pattern transition under different temperature gradients: A phase field study coupled with the parallel adaptive-mesh-refinement algorithm. J. Appl. Phys., 2017, 121(12): 125101.

[11] Medvedev D, Kassner K. Lattice Boltzmann scheme for crystal growth in external flows. Phys. Rev. E, 2005, 72(5 Pt 2): 56703.

[12] Sun D, Zhu M, Pan S, et al. Lattice Boltzmann modeling of dendritic growth in a forced melt convection. Acta Mater., 2009, 57(6): 1755-1767.

[13] Rojas R, Takaki T, Ohno M. A phase-field-lattice Boltzmann method for modeling motion and growth of a dendrite for binary alloy solidification in the presence of melt convection. J. Comput. Phys., 2015, 298: 29-40.

[14] Gyoon Kim S, Tae Kim W, Suzuki T, et al. Phase-field modeling of eutectic solidification. J. Cryst. Growth, 2004, 261(1): 135158.

[15] Raabe D. Overview of the lattice Boltzmann method for nanoand microscale fluid dynamics in materials science and engineering. Modelling Simul. Mater. Sci. Eng., 2004, 12(6): R13-R46.

[16] Tong X, Beckermann C, Karma A, et al. Phase-field simulations of dendritic crystal growth in a forced flow. Phys. Rev. E, 2001, 63(6 Pt 1): 61601.

[17] Beckermann C, Diepers H J, Steinbach I, et al. Modeling melt convection in phase-field simulations of solidification. J. Comput. Phys., 1999, 154(2): 468-496.

[18] Takaki T, Rojas R, Sakane S, et al. Phase-field-lattice Boltzmann studies for dendritic growth with natural convection. J. Cryst. Growth, 2017, 474(15): 146-153.

[19] Gündüz M, Hunt J D. The measurement of solid-liquid surface energies in the $\mathrm{Al}-\mathrm{Cu}, \mathrm{Al}-\mathrm{Si}$ and $\mathrm{Pb}-\mathrm{Sn}$ systems. Acta Mater., 1985, 33(9): 1651-1672.

[20] Maraşli N, Hunt J D. Solid-liquid surface energies in the Al$\mathrm{CuAl}_{2}, \mathrm{Al}^{-\mathrm{NiAl}_{3}}$ and Al-Ti systems. Acta Mater., 1996, 44(3): 1085-1096.

[21] Cao Y F, Chen Y, Li D Z, et al. Comparison of Channel Segregation Formation in Model Alloys and Steels via Numerical Simulations. Metall. Mater. Trans. A, 2016, 47(6): 2927-2939.

[22] Datye V, Langer J S. Stability of thin lamellar eutectic growth. Phys. Rev. B. 1981, 24(8): 4155-4169.

[23] Lee J H, Liu S, Trivedi R. The effect of fluid flow on eutectic growth. Metall. Mater. Trans. A, 2005, 36(11): 3111-3125.

This work was financially supported by the National Natural Science Foundation of China [grant number U1537202], the TsinghuaGeneral Motor International collaboration project [grant number 20153000354], and the UK Royal Society through the Newton International Fellowship Scheme. The authors would also like to thank the National Laboratory for Information Science and Technology in Tsinghua University for access to supercomputing facilities. 